\title{
Comparison between the Efficacies of Clotrimazole in the Treatment of Both Forms of Tinea Versicolor
}

A.I.El-Taweel, R.M.Salem and N.M.Amen

Dermatology, Venereology and Andrology Dept., Faculty of Medicine, Benha Univ., Benha, Egypt

E-Mail: drnesma_mahdy@yahoo.com.

\begin{abstract}
Tinea versicolor is a common skin disorder seen in the daily dermatological practice. Tinea versicolor is an endogenous opportunistic infection caused by Malassezia yeasts which are part of the normal flora that normally live on the skin. Several systemic and topical treatments have been used in the treatment of tinea versicolor but their use has been limited by their side effects or variable therapeutic response. Therefore, there is ongoing search for safer and more effective drugs. In this study, it was aimed to evaluate the safety and the efficacy of topical clotrimazole in the treatment of tinea versicolor. The study included 20 patients of tinea versicolor (10 hypo- and 10 hyperpigmented TVC) who were treated with clotrimazole $0.1 \%$ cream. All patients were selected from the outpatient clinics of Dermatology and Andrology Department of Benha University Hospitals. From this study we can conclude that clotrimazole is equally effective in both forms of tinea versicolor.
\end{abstract}

Keywords: Tinea Versicolor, Clotrimazole.

\section{Introduction}

Tinea versicolor (TVC) is a benign superficial fungal skin infection caused by Malassezia yeasts, which are part of the many microscopic organisms that normally live on the skin. Tinea versicolor is not contagious; rather, it is an endogenous opportunistic infection characterized by multiple hypopigmented and hyperpigmented macules; these may coalesce into large irregular patches and have a fine scaly appearance. Although the lesions may be symptomatic, some patients complain of pruritus and tingling sensation. Meanwhile, in a majority of patients, the lesions are disfiguring, embarrassing and restrict choice of clothing [1].

Several modalities have been used in the treatment of tinea versicolor either topical or systemic antifungals but not all the available treatments are appropriate for all patients as oral antifungal drugs have multiple harmful side effects like fatigue, headache, nausea, vomiting, abdominal pain, diarrhea, skin rashes and liver toxicity. Systemic antifungal drugs should be avoided in pregnancy due to teratogenicity in animals. Also some topical agents can cause skin rash, irritation, mild burning sensation, itching and skin redness; therefore there is an ongoing search for safer and more effective drugs [2].

Clotrimazole is an imidazole antifungal with a broadspectrum fungistatic activity. It inhibits the enzyme lanosterol 14-alpha-demethylase, a cytochrome P 450 dependent enzyme that converts lanosterol to ergosterol blocking the synthesis of ergosterol which is the primary cell sterol of fungi leading to alteration of the cell membrane permeability. Also, it induces changes in oxidative and peroxidative enzyme activities and toxic concentrations of hydrogen peroxide develop intracellular. The overall effect is cell membrane and internal organelle disruption and cell death [3].

\section{Aim of study}

In this study, it was aimed to evaluate the safety and the efficacy of topical clotrimazole in the treatment of both forms of tinea versicolor.
This is a comparative therapeutic study. The study included sixty patients with tinea versicolor attending the dermatology outpatient clinic at Benha University Hospital, Benha, Egypt.

Participants gave their informed written consent before enrollment in the study and the study was approved by the Research Ethics Committee in Faculty of Medicine, Benha University.

All patients included in the study had clinically the typical pityriasis versicolor lesions. Patients with Local inflammatory or infectious diseases at the site to be treated or who were using systemic or topical therapy for TVC during the month preceding the study or who were pregnant or lactating were excluded.

All patients were subjected to a complete clinical examination to evaluate the clinical type and the extent of the disease and a complete wood's light examination to confirm diagnosis through showing yellow fluorescence of the lesions, also to determine the site and extent of the lesions.

The twenty patients were treated with clotrimazole $1 \%$ cream. It applied as a thin layer topically twice daily for 1 month on the affected skin and normal skin around.

The treatment preparation used in this work was commercially available.

The patients were re-evaluated at 2 and 4 weeks from starting the treatment and they were followed up for 1 month after stopping the treatment through clinical and wood's light examinations to report clinical responses, disease recurrence and any local or systemic side effects.

The clinical responses were categorized into complete response, partial response and failure of response.

\subsection{Statistical analysis}

Statistical analysis was performed using Statistical package for Social Science (IBM Corp. Released 2017. IBM SPSS Statistics for Windows, Version 25.0. Armonk, NY: IBM Corp.). Mean \pm SD, Student $T$, one way analysis of variance, Chi-Square, Fisher's exact, Regression analysis were used.

\section{Patients and methods}




\section{Results}

Demographic Data

There was insignificant difference between the studied groups regarding age $(\mathrm{p}=0.654)$ and gender $(\mathrm{p}=0.121)$ Table (1).

Table (1) Demography of all studied cases.

\begin{tabular}{lccc}
\hline & & \multicolumn{2}{c}{ Clotrimazole } \\
\cline { 3 - 4 } & & \multicolumn{2}{c}{$\mathbf{N = 2 0}$} \\
\hline Age & mean \pm SD & 32.1 & \pm 9.7 \\
Male & N \% & 19 & $95 \%$ \\
Female & N \% & 1 & $5 \%$ \\
\hline
\end{tabular}

SD: standard deviation.

There was also insignificant difference between patients with hypo- and hyper-pigmented tinea versicolor regarding age $(\mathrm{p}=0.274)$ and gender $(\mathrm{p}=0.076)$. There was insignificant difference between the studied groups regarding history findings.

There was insignificant difference between the response of hypo- and hyperpigmented TVC to clotrimazole treatment Table (2).

Table (2) Comparison of clinical response and wood's light examination according to skin lesion color after clotrimazole treatment.

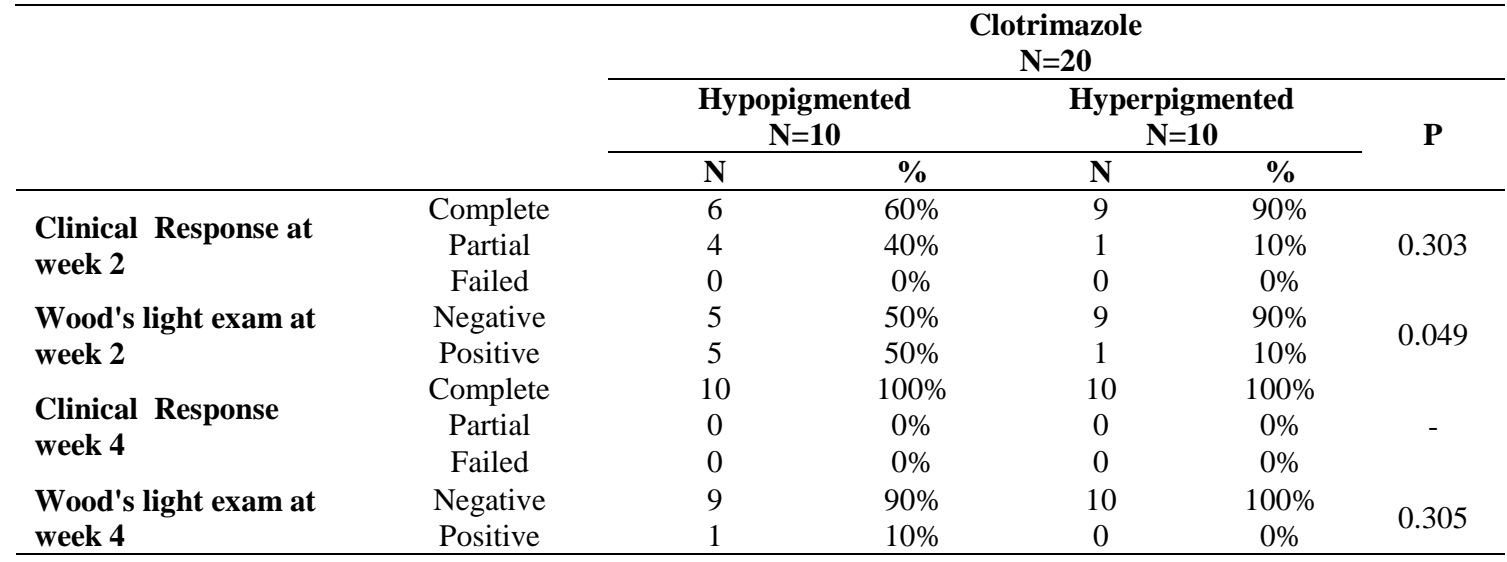

\section{Discussion}

Tinea versicolor is a common fungal infection of the skin caused by Malassezia furfur yeasts which are normal skin flora. They are characterized by multiple hypopigmented and hyperpigmented macules or patches. Tinea versicolor is majorly asymptomatic but cosmetically not acceptable. Different modalities have been used in the treatment of tinea versicolor either topically or systemically but not all the treatments are appropriate for all patients as they have multiple side effects. Therefore, there is an ongoing search for safer and more effective drugs.

Clotrimazole is a broad-spectrum antifungal drug. It inhibits the enzyme lanosterol 14-alpha-demethylase blocking the synthesis of ergosterol which is the primary cell sterol of fungi leading to alteration of the cell membrane permeability. Also, Clotrimazole induces changes of oxidative and peroxidative enzyme activities and toxic concentrations of hydrogen peroxide develop intracellularly leading to cell membrane and internal organelle disruption and fungal cell death [3].

The study included 20 patients of tinea versicolor who were classified into hypo- and hyperpigmented TV patients.

The 20 patients were treated with clotrimazole $0.1 \%$ cream topically twice daily.

All patients were assessed for the efficacy of clotrimazole cream; there was insignificant difference between the two groups.

Clotrimazole has been proven to play a better role than a single dose $400 \mathrm{mg}$ or weekly dose $150 \mathrm{mg}$ of oral flucanazole and single dose therapy with oral ketaconazole as well, in a comparative study [4]. Also, clinical response after 1 month of treatment with clotrimazole was better than a single dose of fluconazole $400 \mathrm{mg}$ in a double-blind randomized controlled trial [5]. In addition, clotrimazole show better antifungal activity than terbinafin $1 \%$ cream in a clinical trial study [6].

\section{Conclusions}

From this study we can conclude that clotrimazole is an equally effective treatment for both forms of tinea versicolor. 


\section{References}

[1] K. Sánchez, M. Bahena, C. Cedillos, E. Cortés, S. Caire, A. Herrera, M. García, Pityriasis Versicolor in Children and Adolescents: an Update. J., Current Fungal Infection Reports, Vol.13(1) PP.157-168, 2019.

[2] A. Gupta, K. Foley, Treatment for Tinea Versicolor. J., Fungi, Vol.1(1), PP. 13-29, 2015.

[3] O. El-garhy, An overview of the azoles of interest. Intern J., Current Pharmaceutical Research, Vol.7(1), PP.1-6, 2015.

[4] S. Ravindranath, Pityriasis versicolor: therapeutic efficacy of various regimes of topical $2 \%$ clotrimazole cream, oral flucanazole and ketoconazole.
International J., Contemporary Medical Research, Vol.3(8), PP.2355-2360, 2016.

[5] M. Dehghan, N. Akbari, N. Alborzi, S. Sadani, A. Keshtkar, Single-dose oral fluconazole versus topical clotrimazole in patients with pityriasis versicolor: A double-blind randomized controlled trial. J., Dermatology, Vol. 37(8), PP.699-702, 2010.

[6] M. Rezvani, S. Sefidgar, Efficacy of Terbinafine $1 \%$ Cream versus Clotrimazole $1 \%$ Cream in The Treatment Of Pityriasis Versicolor. J., Babol University of Medical Sciences, Vol.8(30), PP.42- 46, 2006. 